\title{
Patterns and prognosis of Ventriculoperitoneal shunt malfunction among pediatrics in Saudi Arabia
}

Ikblass S. Altwejri, MBBS, FRCSC, Khulood K. AlRaddadi, MD, Ghadeer A. Alsager, MD, Abdulaziz H. Abobotain, MD, Hissah K. Al Abdulsalam, MD, Salman M. AlQazlan, MD, Nasser A. Almujaiwel, MD.

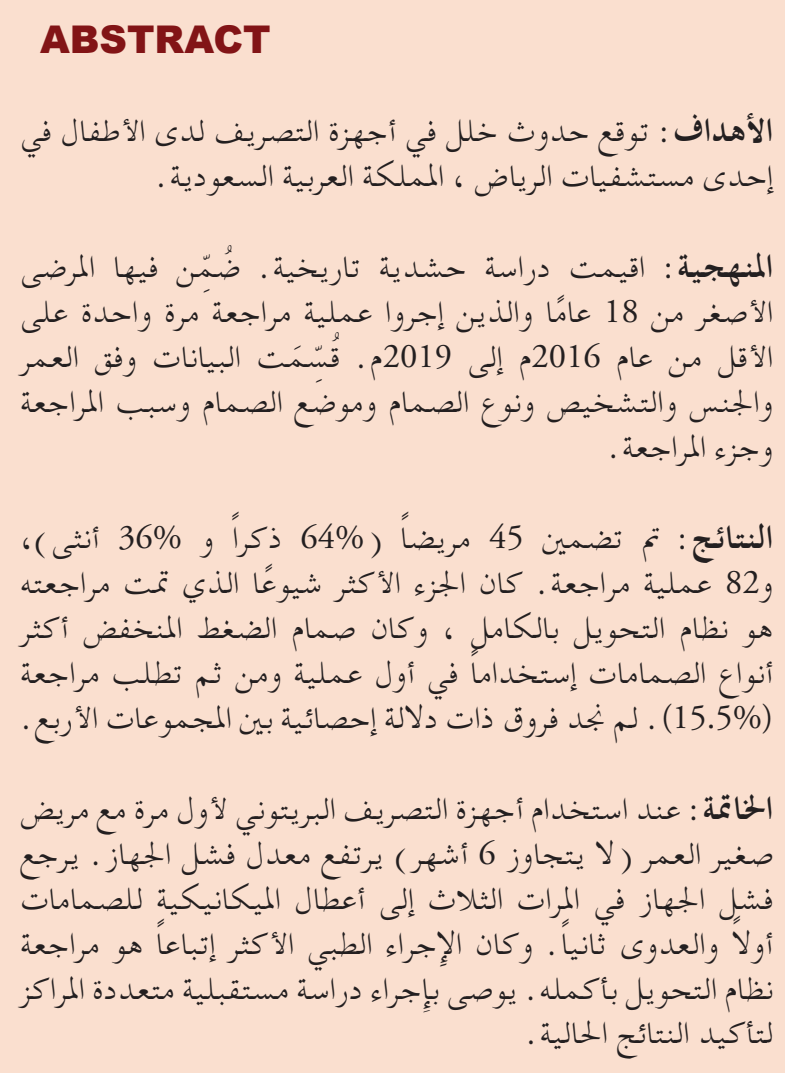

Objectives: To examine the predictors of pediatric ventriculoperitoneal (VP) shunt malfunction in a university hospital.

Methods: A retrospective cohort was conducted. Patients under 18 years old who underwent VP shunt revision at least once between 2016 and 2019 were included. Data were stratified based on age, gender, diagnosis, type of valve, valve position, cause of revision, and part revised.

Results: A total of 45 patients (64\% males and 36\% females) were included in this study. Eighty-two revision surgeries were identified. The most common revised part was the entire shunt system. The most common type of valve which required revision was the low-pressure valve (15.5\%). Since a $p$-value of less than 0.05 was considered significant, no significant differences among the 4 groups for different points.

Conclusions: Younger age at initial VP shunt insertion is associated with a higher rate of shunt malfunction. Valve mechanical failures followed by infections are the most common causes for the first 3 revisions. A prospective multi-center study to confirm the current findings is recommended.

\section{Neurosciences 2020; Vol. 25 (5): 356-361 doi: $10.17712 / n s j .2020 .5 .20200038$}

From the Division of Neurosurgery (Altwejri, Al Abdulsalam), Department of Surgery, College of Medicine, King Saud University, National Neuroscience Institute (AlRaddadi), King Fahad Medical City, College of Medicine (Alsager, AlQazlan, Almujaiwel), King Saud University. Riyadh, Kingdom of Saudi Arabia, and Montreal Neurological Hospital (Abobotain), Montreal, Canada

Received 15th March 2020. Accepted 24th June 2020.

Address correspondence and reprint request to: Dr. Ikblass S. Altwejri, Division of Neurosurgery, Department of Surgery, King Saud University, College of Medicine and King Khalid University Hospital, Riyadh, Kingdom of Saudi Arabia. E-mail: Toikhlass@gmail.com ORCID ID: https://orcid.org/0000-0001-5983-6855

$\mathrm{V}$ entriculoperitoneal (VP) shunt insertion is one of the most common procedures in pediatric neurosurgery for treating hydrocephalus. ${ }^{1}$ Among pediatric age group hydrocephalus is considered a common, surgically correctable condition in which there is an increase in the volume cerebrospinal fluid (CSF), leading to cerebral ventricles dilatation, thinning of the cerebral mantle, and elevation of intracranial pressure. ${ }^{2}$ Patients with VP shunts represent more than 30,000 hospital admissions per year. ${ }^{2}$ Although VP shunting has decreased the morbidity and mortality of hydrocephalus, it is still associated with multiple complications, many of which require surgical revision. ${ }^{1-4}$ Recent studies have reported the rate of 
complications following VP shunting to be between $30 \%$ and $50 \% .^{3-6}$ Shunt malfunctions causes can be categorized into infectious, mechanical, or functional. ${ }^{2-4}$ Shunt failure definition is revision or replacement of the original shunt between 30-days and 1-year. ${ }^{2}$ Mechanical failure can happen at the proximal end, valve, or distal end, and it includes obstruction, disconnection, fracture, distal end migration, or inflammation. Functional failure happens when there is overdrainage or underdrainage of cerebrospinal fluid while the whole shunt system is properly functioning. Infection happens when the patients demonstrate clinical findings and positive fluid sampling results. ${ }^{3}$ The most common complication necessitating revision are obstruction, infection, and displacement. ${ }^{2,3,6,7}$ Shunt failure is most common within the first 2 years postoperatively. ${ }^{3}$ Many important predictors of VP shunt failure have been reported in the literature, including etiologies of the hydrocephalus, prematurity, and age at the initial placement of the shunt. ${ }^{1,8,9}$ Clinical factors that may increase the risk of shunt revision include time from the first surgery, surgical procedure duration, prior treatments, number of surgeons, surgical expertise, surgical technique, anatomic site of the shunt, and diagnosis. ${ }^{1,-10}$ Proximal catheter tip location is another predictor, as tip positions in the Foramen of Monro, lateral ventricles, or the third ventricle all were associated with lower rates of surgical revision. ${ }^{11}$ Other predictors of shunt failure were reported to be poor catheter placement and use of a non-programmable valve. ${ }^{12}$

Previous studies have emphasized the need to identify children at risk, and for prospective cohorts to to investigate the relationship of risk factors and incidence of shunt revisions. ${ }^{1,9,10}$ Risk factors for VP shunt complications vary across institutions and populations, and a very limited number of studies addressing VP shunt malfunction have been conducted in Saudi Arabia, and only one in the last 10 years was published. ${ }^{13}$

The goal of this study was to determine patterns and predictors of pediatric VP shunt malfunction, causes of the hydrocephalus and VP shunt revision and type of valve malfunction and define the best preventive measures. This knowledge will contribute to lowering the incidence of shunt malfunction, decreasing the

Disclosure. Authors have no conflict of interests, and the work was not supported or funded by any drug company. number of surgeries, and increasing complication-free intervals between surgeries in hydrocephalic pediatric patients.

Methods. Database creation and literature review. The study was carried out using a retrospective cohort design. Patients below the age of 18 admitted to the neurosurgery ward at King Saud University Medical City for revision of VP shunt from February 2016 to February 2019 were with complete medical records included. The exclusion criteria were conservative, nonsurgical management, bilateral VP shunts, VP shunt revision at other institutions, age above 18 years at initial insertion or subsequent revisions, and missed or incomplete documentation.

Information about the revision procedures was collected from the patients' medical records. A standardized, comprehensive data collection sheet was used to collect patient data, including sociodemographic information, cause of hydrocephalus, date of diagnosis, type of shunt system used, shunt insertion technique, number of previous revisions, and treatment of the primary pathology.

Patients were stratified by age ( $<6$ months, $>6$ months, 9-12 months, 1-3 years, 3-6 years, $>6$ years), gender (male and female), diagnosis (congenital hydrocephalus, intracranial tumor, myelomeningocele, aqueductal stenosis, post-traumatic, and post-infectious), hole placement (frontal vs occipital), type of valve (low, medium, high pressure, or programmable), valve position (burr hole: old or new at the revision, in-line), the reason for revision (shunt infection, proximal catheter disconnection, proximal catheter obstruction, distal catheter obstruction, valve mechanical failure, and others), and part revised (whole, proximal, and distal).

The strategies for the literature search were conducted across PUBMED/MEDLINE using the following MeSH search terms: Ventriculoperitoneal Shunt; Pediatrics; Malfunction; Shunt. Searches were tailored using the Boolean operators "AND or OR". Reference lists of reviews and retrieved articles were

Table 1 - Demographic distribution of patients

\begin{tabular}{lc}
\hline Etiology & $\mathbf{n}(\%)$ \\
\hline Congenital & $17(38)$ \\
Myelomeningocele & $14(31)$ \\
Intracranial tumor & $4(9)$ \\
Post-infection & $2(4)$ \\
Post-trauma & $1(2)$ \\
Others & $7(16)$ \\
\hline
\end{tabular}


assessed for further studies.

Variables and definitions. In our project, shunt system dysfunction was divided into proximal end dysfunction, valve dysfunction, distal end dysfunction, or the entire system dysfunction. Possible causes of revision are mechanical shunt failure or infection.

Mechanical shunt failure was defined by any of the following situations: obstruction of any part by blood, debris, choroid plexus or tissue parenchyma, kinking, adhesions, disconnection, fracture, distal end migration confirmed radiologically or intraoperatively by the surgeon.

Infection was defined by positive clinical findings: high temperature upon presentation, high white blood cell count, or fluid sampling results.

Statistical analysis. Primary variables assessed included age, gender, diagnosis, type of valve, the reason for revision, and part revised. The Kruskall-Wallis test was used to assess the median number of replacements across different groups. No post hoc tests were used due to the $p$-value indicating no significant relation Data analysis was carried out using IBM SPSS statistical software version 22.0. A $p$-value $<0.05$ was considered statistically significant.

Ethical aspects. The hospital ethics committee approved the study protocol. There was no need for informed consent for participation in the study as each participant was represented in the database using an anonymous unique identifier (ID).

Results. A total of 137 patients underwent VP shunt placement at our hospital during the period of this study. These consisted of 80 adults and 57 pediatric cases. Amongst the pediatric cases, 45 had a complete

Table 2 - Causes of Ventriculoperitoneal Shunt revision $(n=45)$.

\begin{tabular}{lccccc}
\hline Cause of revision & $\begin{array}{c}\text { Total cases at first } \\
\text { revision }(\mathbf{n}=45)\end{array}$ & $\begin{array}{c}\text { Total cases at } \\
\text { second revision } \\
(\mathbf{n}=\mathbf{2 1})\end{array}$ & $\begin{array}{c}\text { Total cases at third } \\
\text { revision } \\
(\mathbf{n}=11)\end{array}$ & $\begin{array}{c}\text { Total cases at } \\
\text { fourth revision } \\
(\mathbf{n}=5)\end{array}$ & $P$-value \\
\cline { 2 - 4 } & \multicolumn{2}{c}{$\mathbf{n}(\%)$} \\
\hline Valve mechanical failure & $15(33)$ & $4(19)$ & $4(36)$ & $1(20)$ & 0.595 \\
Shunt infection & $11(24)$ & $8(38)$ & $3(27)$ & $2(40)$ & 0.660 \\
Proximal catheter malfunction & $8(18)$ & $2(9.5)$ & $1(9)$ & $0(0)$ & 0.580 \\
Distal catheter obstruction & $5(11)$ & $2(9.5)$ & $0(0)$ & $1(20)$ & 0.598 \\
Others & $3(7)$ & $5(24)$ & $3(27)$ & $1(20)$ & 0.156 \\
*Not documented & $3(7)$ & $0(0)$ & $0(0)$ & $0(0)$ & 0.464 \\
\hline
\end{tabular}

Table 3 - Revised part during shunt revisions

\begin{tabular}{lccccc}
\hline $\begin{array}{l}\text { Surgical procedures done } \\
\text { during shunt revisions }\end{array}$ & $\begin{array}{c}\text { Total cases at } \\
\text { first revision } \\
(\mathbf{n}=\mathbf{4 5})\end{array}$ & $\begin{array}{c}\text { Total cases at } \\
\text { second revision } \\
(\mathbf{n}=\mathbf{2 1})\end{array}$ & $\begin{array}{c}\text { Total cases at third } \\
\text { revision } \\
(\mathbf{n}=11)\end{array}$ & $\begin{array}{c}\text { Total cases at } \\
\text { fourth revision } \\
(\mathbf{n}=5)\end{array}$ & $P$-value \\
\hline Distal catheter & $4(9)$ & $4(19)$ & $1(9)$ & $0(0)$ & 0.521 \\
Proximal + valve & $6(13)$ & $0(0)$ & $0(0)$ & $0(0)$ & 0.150 \\
Proximal catheter & $7(16)$ & $3(14)$ & $2(18)$ & $0(0)$ & 0.801 \\
Valve & $9(20)$ & $3(14)$ & $4(36)$ & $0(0)$ & 0.311 \\
Whole shunt system & $17(38)$ & $10(48)$ & $4(36)$ & $4(80)$ & 0.298 \\
Not documented & $2(4)$ & $1(5)$ & $0(0)$ & $1(20)$ & 0.490 \\
\hline
\end{tabular}

medical record and underwent VP shunt revision and thus were included in the final analysis.

A total of 45 pediatric patients $(64 \%$ males and $36 \%$ females), with a total of 82 revision surgeries were included in the study. The underlying causes of hydrocephalus in the selected sample are shown in Table 1.

All the included patients required one or more shunt
Table 4 - Median number of revisions across different valve types.

\begin{tabular}{lccc}
\hline Valve type & $\begin{array}{c}\text { Median number } \\
\text { of revisions }\end{array}$ & $\begin{array}{c}\text { Interquartile } \\
\text { range }\end{array}$ & P-value \\
\hline Low pressure & 1 & 1 & 0.773 \\
Medium pressure & 1.5 & 2 & \\
Medium-low pressure & 1 & 1 & \\
Programmable & 2 & 3 & \\
\hline
\end{tabular}


Table 5 - Hole placement in revision surgery.

\begin{tabular}{lcccc}
\hline Hole locations & $\begin{array}{c}\text { Total cases at first } \\
\text { revision }(\mathbf{n}=\mathbf{4 5})\end{array}$ & $\begin{array}{c}\text { Total cases at second } \\
\text { revision }(\mathbf{n}=\mathbf{2 1})\end{array}$ & $\begin{array}{c}\text { Total cases at third } \\
\text { revision }(\mathbf{n}=11)\end{array}$ & $\begin{array}{c}\text { Total cases at fourth } \\
\text { revision }(\mathbf{n}=5)\end{array}$ \\
\hline Same hole placement & $29(33)$ & $18(85.7)$ & $9(81.8)$ & $3(60)$ \\
New hole placement & $12(26.6)$ & $3(14.3)$ & $2(18.2)$ & $1(20)$ \\
Not documented & $4(8.8)$ & $0(0)$ & $0(0)$ & $1(20)$ \\
\hline
\end{tabular}

revisions, 45 (100\%). Twenty-one (47\%) required revision twice, $11(24 \%)$ required revision 3 times, and $5(11 \%)$ required revision 4 times.

Among the sample, 52\% (17\% female, 35\% male) failed VP shunting within 24 months after initial placement and required revision surgery. The most common age group requiring more than 2 revision surgeries was under 6 months, followed by 1-3 years of age. The causes of VP shunt revisions are illustrated in Table 2.

In our sample, the most common surgical revisions involved revising the entire shunt system. The frequency of replaced parts during shunt revisions appears in Table 3. The most common type of valve used at initial insertion was low-pressure (33\%), followed by medium-pressure (27\%), programmable (20\%), and medium-low (13\%).

The most common valve requiring revision surgery was the low-pressure valve (15.5\%).
We can see from Table 4 and Figure 1 that low and medium-low pressure valves having one median replacement and medium and programmable valves have a slightly higher number of median revisions. However, this was not shown to be statistically significant. Burr hole location in each revision surgery illustrated in Table 5.

Discussion. Hydrocephalus is a commonly encountered disease in pediatric neurosurgery, managed with VP shunt insertion. ${ }^{1,2}$ Shunt complications necessitating revisions are common, with revision rates ranging from $30 \%$ to $50 \%$. $^{3-6}$ This study included 45 patients with 82 revision surgeries. The most common age group requiring multiple revision surgeries was under 6 months. In the following section, we will be comparing and contrasting our findings with what is reported in the literature.

Many indications for ventriculoperitoneal shunt

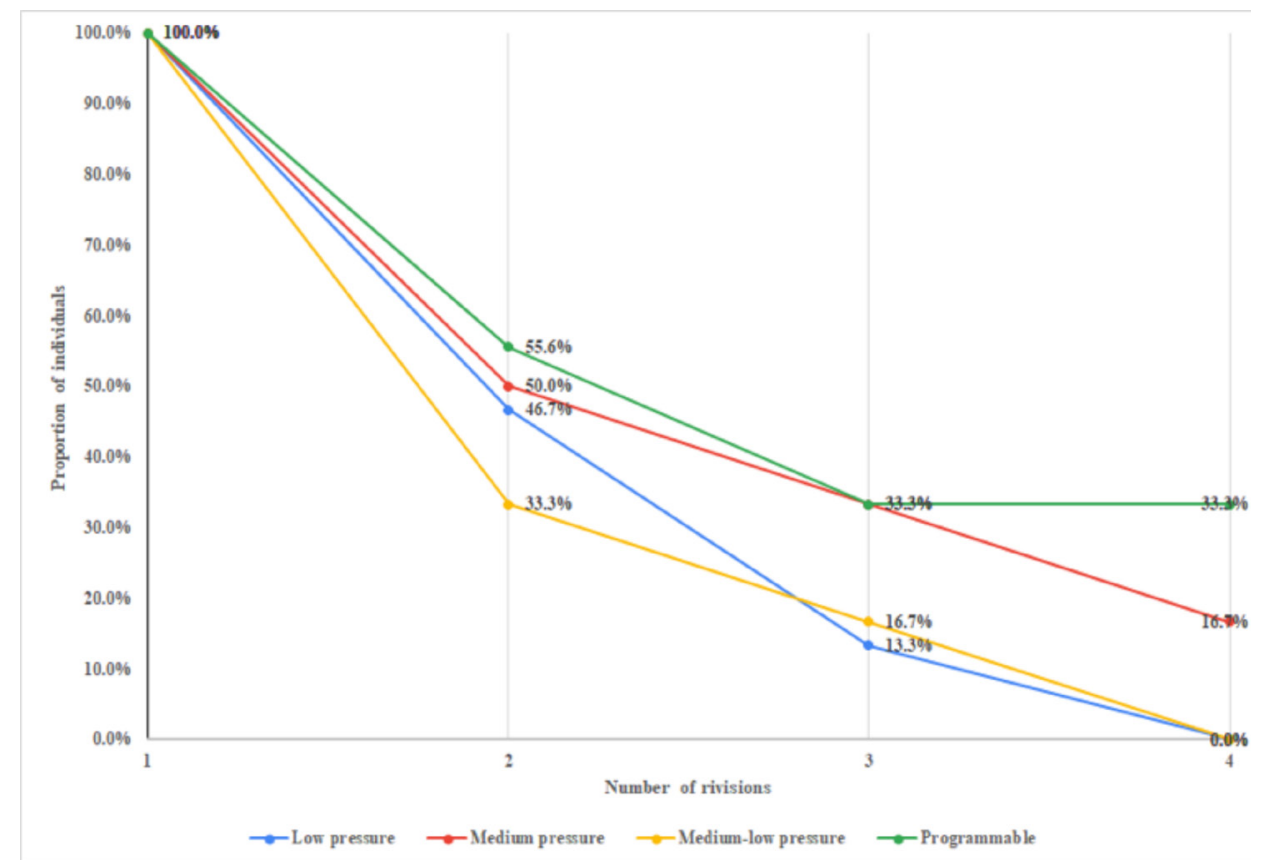

Figure 1 - Proportion of individuals underwent the valve revisions and corresponding cumulative valve revision frequencies, stratified according to the valve type 
revision surgeries have been reported. The most common cause is often mechanical failure, although the specific cause differs, and most studies report the second most common is infection. We report similar results, where valve mechanical failure and infections were found to be the most common indications for the first 3 revisions, with valve mechanical failure accounting for $33 \%, 19 \%$, and $36 \%$ of the first three revisions, respectively. Infection accounted for $24 \%, 38 \%$, and $27 \%$ of the first three, respectively.

Pan $\mathrm{P}^{6}$ retrospectively evaluated risk factors leading to shunt failures in 137 hydrocephalus patients. The most common complications in the cohort were shunt blockade (45.94\%), and shunt infection (16.21\%). Ahmadvand $S$, et $\mathrm{al}^{7}$ retrospectively studied 82 patients with 121 revisions, the most common complication reported was obstruction of the shunts (53.6\%). Agarwal $\mathrm{N}$ et $\mathrm{al}^{9}$ retrospectively studied complications of VP shunt in 41 hydrocephalus patients and 47 complications. Mechanical complications occurred in $33.33 \%$, followed by infective complications (15.63\%). Almazroea, A study ${ }^{13}$ from Saudi Arabia retrospectively studied shunt complications in 65 pediatric patients. Obstruction was the most common cause $38.46 \%$ followed by infection $12.3 \%$.

The high probability of failure among the younger age group has been reported by several studies and matches our findings, where we found that the most common age group requiring more than 2 revision surgeries was under 6 months old. Agarwal $\mathrm{N}$ et al ${ }^{9}$ and Tervonen $\mathrm{J}^{14}$ et al studies both reported that the majority of patients who had shunt failure were operated on in the first 2 years of life.

Ahmadvand $S$ et $\mathrm{al}^{7}$ retrospectively studied 30 -day shunt failure rate in 82 patients $71 \%$ of whom belonged to the pediatrics age group with 121 revisions. The following risk factors were studied: age, gender, household income, level of education, cause of hydrocephalus, causes of revision, type of failure, anatomic site, duration of operation, time of surgery, surgeons' level of expertise, and Glasgow Coma Scale (GCS) score. Their univariate analysis reported that gender, age, anatomic site, GCS score, surgeons' level of expertise, and cause of hydrocephalus were significant factors. they also reported proximal catheter to be the main type of shunt failure (70.0\%). While our results reported the whole shunt system followed by the valve to be the main types of failure.

Venable GT et $\mathrm{al}^{15}$ reviewed 525 patients with 927 shunt insertion or revision surgeries. They studied risk factors comparing preventable and non-preventable causes. The most common causes for both groups of shunt revision $(\mathrm{n}=202)$ were proximal catheter failure $41.6 \%$, followed by valve dysfunction $17.3 \%$, and distal catheter malfunction $13.4 \%$. We report different results where valve mechanical failure and infections were the most common indications for the first 3 revisions, followed by proximal catheter malfunction, accounting for $33 \%$ and $24 \%$, and $18 \%$ respectively on the first revision.

The most common cause for initial shunt insertion in our study was congenital hydrocephalus in the age group of 0-12 months (38\%). Our results are comparable to what was previously reported, where it was reported that congenital hydrocephalus was the most common cause with its rate ranging from $36.5 \%-47 \% .^{6,7}$

Our study was limited by its retrospective nature and the small sample size, as only patients with a VP shunt revision in our institution with complete detailed documentation in a single institute. We recommend a multi-center study with a larger sample size to confirm the current findings.

In conclusion, higher rate of shunt failure was noted in pediatrics with younger age at initial VP shunt insertion (below 6 months). Valve mechanical failure followed by infections were the most common causes in the first 3 revisions. Revision of the entire shunt system was the most commonly performed procedure 38\%. Our results were not consistent with the literature which showed revision of peritoneal (distal) part of the shunt as the most commonly performed procedure. A multicenter study to increase the sample size and confirm the current findings in Saudi Arabia is recommended.

Acknowledgment. We would like to thank PageCure (https://www.pagecure.com/) for English language editing.

\section{References}

1. Erps A, Roth J, Constantini S, Lerner-Geva L, Grisaru-Soen G. Risk factors and epidemiology of pediatric ventriculoperitoneal shunt infection. Pediatr Int (Roma) 2018; 60: 1056-1061.

2. Wright Z, Larrew TW, Eskandari R. Pediatric Hydrocephalus: Current State of Diagnosis and Treatment. Pediatr Rev 2016; 37: 478-490.

3. Bates P, Rajderkar D. Common and Uncommon Causes of Ventriculoperitoneal Shunt Malfunction Diagnosed on Plain Radiographs. Curr Probl Diagn Radiol 2018; 47: 317-323.

4. Hanak BW, Bonow RH, Harris CA, Browd SR. Cerebrospinal Fluid Shunting Complications in Children. Pediatr Neurosurg 2017; 52: 381-400.

5. Abode-Iyamah KO, Khanna R, Rasmussen ZD, Flouty O, Dahdaleh NS, Greenlee J, et al. Risk factors associated with distal catheter migration following ventriculoperitoneal shunt placement. J Clin Neurosci 2016; 25: 46-49. 
6. Pan P. Outcome Analysis of Ventriculoperitoneal Shunt Surgery in Pediatric Hydrocephalus. J Pediatr Neurosci 2018; 13: 176-181.

7. Ahmadvand S, Dayyani M, Etemadrezaie H, Ghorbanpour A, Zarei R, Shahriyari A, et al. Rate and Risk Factors of Early Ventriculoperitoneal Shunt Revision: A Five-Year Retrospective Analysis of a Referral Center. World Neurosurg 2020; 134 : e505-e511.

8. Yakut N, Soysal A, Kepenekli Kadayifci E, Dalgic N, Yılmaz Ciftdogan D, Karaaslan A, et al. Ventriculoperitoneal shunt infections and re-infections in children: a multicentre retrospective study. Br J Neurosurg 2018; 32: 196-200.

9. Agarwal N, Shukla RM, Agarwal D, Gupta K, Luthra R, Gupta J, et al. Pediatric Ventriculoperitoneal Shunts and their Complications: an Analysis. J Indian Assoc Pediatr Surg 2017; 22: $155-157$.

10. Anderson IA, Saukila LF, Robins JM, Akhunbay-Fudge CY, Goodden JR, Tyagi AK, et al. Factors associated with 30-day ventriculoperitoneal shunt failure in pediatric and adult patients. J Neurosurg 2018; 130: 145-153.
11. Dobran M, Nasi D, Mancini F, Gladi M, Polonara G, Marini A, et al. Relationship between the location of the ventricular catheter tip and the ventriculoperitoneal shunt malfunction. Clin Neurol Neurosurg 2018; 175: 50-53.

12. Jeremiah KJ, Cherry CL, Wan KR, Toy JA, Wolfe R, Danks RA. Choice of valve type and poor ventricular catheter placement: Modifiable factors associated with ventriculoperitoneal shunt failure. J Clin Neurosci 2016; 27: 95-98.

13. Almazroea A. Epidemiology of ventriculoperitoneal shunt complications in pediatric age group in medina region: Observational study. Clin Med Invest 2019; 4: 1-3.

14. Tervonen J, Leinonen V, Jääskeläinen JE, Koponen $S$, Huttunen TJ. Rate and Risk Factors for Shunt Revision in Pediatric Patients with Hydrocephalus-A Population-Based Study. World Neurosurg 2017; 101: 615-622.

15. Venable GT, Rossi NB, Morgan Jones G, Khan NR, Smalley ZS, Roberts ML, et al. The Preventable Shunt Revision Rate: a potential quality metric for pediatric shunt surgery. J Neurosurg Pediatr 2016; 18: 7-15.

\section{Supplements}

* Supplements will be considered for work including proceedings of conferences or subject matter covering an important topic

* Material can be in the form of original work or abstracts.

* Material in supplements will be for the purpose of teaching rather than research.

* The Guest Editor will ensure that the financial cost of production of the supplement is covered.

* Supplements will be distributed with the regular issue of the journal but further copies can be ordered upon request.

* Material will be made available on Saudi Medical Journal website 